\title{
ARTE, ENTORNO, SOSTENIBILIDAD. APROVECHAMIENTO DE RESIDUOS LÍTICOS PROVENIENTES DE AGUAS SUBTERRÁNEAS CARBONATADAS*
}

\author{
M. Isabel Sánchez Bonilla, Tomás de A. Oropesa Hernández, \\ Mauricio Pérez Jiménez, Francisco J. Viña Rodríguez, Cecile Meier, \\ M. ${ }^{a}$ Fernanda Guitán Garre, Mariano J. Pérez Sánchez y Juan R. Núńez Pestano**
}

\section{RESUMEN}

Se han localizado, analizado y aplicado creativamente residuos minerales que depositan las aguas subterráneas, sobresaturadas en $\mathrm{CO}_{2}$, extraídas mediante galerías/minas. El análisis documental abarca el archipiélago canario; la recogida de muestras y el desarrollo experimental se ha realizado principalmente en galerías y conducciones de Tenerife. Las rocas-residuo de mayor resistencia han demostrado ser magníficos materiales para esculturas labradas en formato medio. Los restos fragmentados y las rocas de menor consistencia, tipo toba, pueden encontrar utilidad en fabricación de cales-morteros compatibles con la restauración de obras patrimoniales. Debido a la capacidad petrificadora de estas aguas, se abren múltiples posibilidades en creación tridimensional mediante moldes y/o madreformas, tanto en escultura (obra única) como en actividades artesanales que requieren repetición sucesiva de elementos de similares características. La recopilación exhaustiva de datos y los análisis efectuados evidencian la necesidad de una investigación básica multidisciplinar que ofrezca, en relación con el tratamiento de las aguas subterráneas y las salmueras de rechazo, alternativas de reutilización compatibles con la sostenibilidad medioambiental.

PAlabras Clave: escultura, entorno volcánico, agua subterránea, roca, toba, travertino.

\section{ART, ENVIRONMENT, SUSTAINABILITY. USE OF LITHIC WASTE FROM CARBONATED GROUNDWATER}

\begin{abstract}
Mineral residues deposited in groundwater have been identified, analyzed and creatively applied. These residues are oversaturated in $\mathrm{CO}_{2}$ and are extracted by means of galleries/ mines. The documentary analysis covers the Canary Archipelago; the collection of samples and experimental development has been carried out mainly in galleries and conducts in Tenerife. The most resistant waste-rocks have proved to be magnificent materials for medium format sculpture. The fragmented remains and the rocks of lesser consistency, such as tufa, can be useful in the manufacture of lime-mortars compatible with the restoration of heritage works. Due to the petrifying capacity of these waters, multiple possibilities are opened in three-dimensional creation by means of molds and/or mother forms, both in sculpture (unique work) and in artisan activities that require successive repetition of elements of similar characteristics. The exhaustive collection of data and the analyses carried out show the need for basic multidisciplinary research that offers, in relation to the treatment of groundwater and reject brine, alternatives for reuse that are compatible with environmental sustainability.

KEYwORDS: sculpture, volcanic environment, groundwater, rock, tuff, travertine.
\end{abstract}




\section{INTRODUCCIÓN}

Como ocurre en otros lugares de origen volcánico, las aguas subterráneas de Canarias se encuentran, en muchas ocasiones, sobresaturadas de $\mathrm{CO}_{2}$, con una mineralización que aumenta progresivamente, ya que tanto el modelo económico tradicional, basado en sucesivos monocultivos para exportación, como el actual, con la recepción de más de catorce millones de turistas al año y mantenimiento de los cultivos de plátano y tomate, han requerido una explotación de los acuíferos que supera ampliamente la recarga. Para cubrir la demanda de agua se han ido implementando y ampliando, de manera sistemática desde hace más de un siglo, las obras de captación: galerías en zonas de medianías y pozos en las zonas costeras.

El objetivo general del proyecto de investigación financiado que resumimos es la aplicación creativa de los «residuos» carbonáticos depositados por las aguas de algunas galerías ${ }^{1}$, mirados como materiales pétreos aptos para procesos de $\operatorname{labra}^{2} \mathrm{y}$, simultáneamente, desde ópticas más atrevidas y novedosas: ensayando procesos de petrificación a partir de moldes en termoplástico (impresión 3D en ácido poliláctico o PLA), cera-parafina, silicona, etc., tanto para obra única como para reproducción múltiple, e indagando sobre nuevas posibilidades formales a las que se puede llegar con madreformas, actuando mediante inmersión, fluido, espray, etc.; nos interesa llegar a obras escultóricas de tamaño pequeño o medio, y también analizar espacios naturales y ensayar maquetas para una intervención ambiental de gran envergadura, que pudiera llegar a ser reclamo turístico, al tiempo que referente en la difusión científica y cultural sobre la formación de tobas y travertinos.

* Proyecto de investigación financiado con fondos propios de la ULL. Presentado en convocatoria competitiva en 2018 (referencia: 2018/001483).

** María Isabel Sánchez Bonilla, ULL (Dpto. Bellas Artes) sbonilla@ull.es; Tomás de Aquino Oropesa Hernández, ULL (Dpto. Bellas Artes) toropesa@ull.es; Mauricio Pérez Jiménez, ULL (Dpto. Bellas Artes), mperjim@ull.es; Francisco Javier Viña Rodríguez, ULL (Dpto. Bellas Artes), franvi@ull.es; Cecile Meier, ULL (Dpto. Bellas Artes), cemeier@ull.es; María Fernanda Guitán Garre, ULL (Dpto. Bellas Artes), fguitian@ull.es; Mariano J. Pérez Sánchez, ULL (Dpto. Química), mjperez@ull.es; Juan Ramón Núñez Pestano, ULL (Dpto. Historia), jrnupe@ull.es.

${ }^{1}$ Se llama galería al túnel, casi horizontal, que permite drenar los acuíferos. Aunque contamos con ejemplos de mayor antigüedad, la perforación sistemática y progresiva se llevó a cabo principalmente a mediados del siglo xx, con reperforaciones periódicas hasta la actualidad, llegando en muchas ocasiones a profundidades cercanas a los seis kilómetros. Muchas de ellas están abandonadas/inactivas, algunas nunca llegaron a drenar cantidades significativas.

2 Dentro del Grupo de Investigación Arte y Entorno de la ULL, una de las líneas en que se viene trabajando desde hace algo más de cinco años ha sido la selección-diseño-labra de estas piedras depositadas por el agua de las galerías. Se presentó la ponencia «Las piedras del agua. Posibilidades escultóricas y propuesta ambiental» en el V Simposio Virtual Internacional Valor y Sugestión del Patrimonio Artístico y Cultural (Universidad de Málaga, 8 a 22 de julio de 2015). Además, se han expuesto obras escultóricas realizadas en los materiales calcáreos objeto de estudio en el Centro Cultural de Guía de Isora (julio 2015), en la Galería Magda Lázaro (noviembre 2105), en la Galería Magda Lázaro (julio 2016) y en EDAR Valle de Guerra (marzo 2019). 
Se ha incluido también en el proyecto una línea específica de estudio/análisis de las características diferenciales que concurren en las cales de Canarias y su aplicabilidad en el ámbito de la restauración de bienes culturales. La idea de cara al futuro es actuar como dos proyectos coordinados.

La investigación experimental se ha centrado en las aguas que muestran mayor tendencia bicarbonatada sódico-cálcica, presentando capacidad petrificante. Para este primer proyecto financiado se han seleccionado puntos diversos de la isla de Tenerife, entorno que con sus cuatrocientas galerías activas, algunas de ellas con aguas muy carbonatadas, incluyendo varias que superan $\operatorname{los} 30^{\circ} \mathrm{C}$ en bocamina, reúne las circunstancias más favorables para la toma de muestras y el trabajo experimental; el acuífero de Tenerife, al estar muy compartimentado por la intrusión de diques, ofrece aguas que pueden llegar a ser muy diferentes entre sí, lo que enriquece esta fase de la investigación, en la que han de quedar fijados los protocolos básicos de actuación para las siguientes islas.

Este proyecto ha permitido también el acercamiento a las estaciones de tratamiento de aguas subterráneas, EDAS, evidenciando la conveniencia de plantear un proyecto multidisciplinar específico dedicado a buscar un mejor aprovechamiento de las salmueras de rechazo.

\section{EL ENTORNO. AGUAS, CALES}

Cada una de las siete islas ofrece un panorama específico en cuanto a incidencia y distribución de las aguas que presentan niveles altos de carbonatos.

La Palma es la única isla que tiene buen balance hídrico, con 120 captaciones activas, que en general aportan aguas de excelente calidad, excepto en la zona sur, donde el volcanismo residual aporta $\mathrm{CO}_{2}$, haciéndolas bicarbonatadas.

En El Hierro, el contenido en carbonatos y bicarbonatos es muy elevado en la mitad oeste (volcanismo de la cabecera de El Golfo), con menor incidencia en el resto de la isla. En La Gomera las captaciones más frecuentes son los pozos; hay, no obstante, cinco galerías activas y en las fichas de cuatro de ellas se avisa peligrosidad por "gases», lo que alerta sobre posible saturación en $\mathrm{CO}_{2}$.

En Tenerife se han perforado más de mil galerías, de las que permanecen activas unas 400 , un porcentaje significativo de ellas con aguas de alta capacidad petrificadora, como se verá en detalle al describir la fase experimental.

Gran Canaria se encuentra realizando un exhaustivo proceso de inventario, en el que ya se han incluido 2754 captaciones, para lo que se han tomado como punto de partida cerca de tres mil expedientes de pozos y dos mil de galerías, la mayoría en desuso. Los bajos niveles del acuífero llevaron, hace casi medio siglo, a limitar el suministro de aguas subterráneas a las medianías, resolviendo mediante almacenamiento masivo de agua de lluvia y desalación de agua de mar la mayoría de las poblaciones costeras y las necesidades asociadas al turismo; cuenta con un centenar de desaladoras.

La isla de Lanzarote fue pionera en la aplicación de técnicas de desalación, instalando la primera planta europea de esta índole en 1964, inicialmente para tra- 
tar aguas provenientes de las galerías de Famara; hoy la isla se abastece casi totalmente mediante desalación de agua de mar, y mantiene activas únicamente dos galerías, ambas en el municipio de Haría.

Fuerteventura, la isla más árida del archipiélago, instaló su primera planta para tratamiento de aguas salobres (Tarajalejo-Hotel Maxorata) hace más de cuarenta años; en la actualidad el 100\% de las necesidades hídricas insulares se cubren mediante técnicas de desalación, aplicadas tanto al agua de mar como a aguas subterráneas. En relación con estas últimas, anotemos que cuenta con unas 25 estaciones desalinizadoras de agua salobre (EDAS), que dan servicio a las explotaciones agrícolas de sus titulares, ubicadas principalmente en el término de Tuineje.

Se ha revisado también la importancia histórica de la cal en Canarias, encontrando algunas especificidades que se irán reseñando a continuación. Destacan Fuerteventura y Lanzarote como exportadores habituales de piedra de cal y, en menor proporción, de polvo ya cocido, al resto de las islas del archipiélago. Una actividad que se inició con la Conquista, perdurando hasta los años sesenta del siglo $\mathrm{xx}^{3}$. Resultan también muy interesantes los datos disponibles sobre Gran Canaria. Anotaremos algún detalle sobre existencia-producción de cal en Tenerife. Sobre el resto de las islas, no tenemos datos que proceda considerar.

Fuerteventura es la mayor exportadora de cal, desde la Conquista hasta el siglo xx. Se han censado más de trecientos hornos. En cuanto a calidades, Hausen (1958) distinguía para esta isla entre las calizas marinas, que ubica cercanas a las costas, y la caliza travertina, cuyo origen explica como consecuencia de la intensa aridificación-tracción capilar del Cuaternario que generó una capa calcárea con grosores variables (en determinados puntos llega a los $10 \mathrm{~m}$ ), de la que se nutren el mayor número de canteras, produciendo cales de calidad: poca retracción, buena adherencia, plasticidad, buen comportamiento térmico y transpirabilidad. También menciona Hausen un afloramiento singular, ubicado en el Llano del Diner, que describe como depósito de incrustación, con la caliza cristalizada como calcita en largas formas prismáticas.

Respecto de Lanzarote, existe constancia de la traída de cal, en el siglo xv, desde Rubicón a Las Palmas de Gran Canaria. Desde comienzos del siglo xvi constan sucesivos envíos a Tenerife y La Palma, con una actividad exportadora que irá progresivamente en aumento. A título de ejemplo, mencionaremos que entre el 29 de abril y el 1 de agosto de 1699, salieron del puerto real de Janubio 22 barcos con

${ }^{3}$ La cal había cubierto durante siglos diferentes funciones: aglutinante de morteros, enfoscado/enjabelgado de paramento, desinfectante. Con el auge de los morteros de cemento tipo portland, desde principios del siglo xx, se van abandonando los morteros de cal. Durante la Segunda Guerra Mundial y la Guerra Civil Espańola el desabastecimiento y encarecimiento de materias primas importadas lleva a activar nuevamente la producción de cal para autoconsumo. Así, por ejemplo, para Telde se reseñan tres hornos funcionando en 1944, siendo más de 15 los activos en 1951. A partir de los años sesenta el abaratamiento del cemento lleva nuevamente a su uso prioritario para morteros. Algo similar ocurre con la introducción de pinturas plásticas y desinfectantes químicos. En la actualidad asistimos a la conservación/restauración con función etnográfica y reclamo turístico. 
piedra de cal (Hernández Delgado y Rodríguez Armas 1993). Conviene recordar, no obstante, que el puerto de Janubio quedó cerrado e inutilizado a causa de las erupciones de Timanfaya (1730-1736), cuyas lavas cubrieron también los territorios caleros ubicados al norte del mismo. Otra zona importante de extracción fue la zona de Órzola, en el norte de la isla, donde se ha podido constatar la existencia de al menos diez caleras.

Gran Canaria, aunque no ha tenido tanta importancia como exportadora de cal, ha contado con material para cubrir sus propias necesidades, explotando para ello confites ${ }^{4}$ y caliches (denominación local de materiales similares a los anotados en Fuerteventura como travertina). Se citan a nivel histórico yacimientos y hornos en múltiples lugares: El Calero en Telde, Tamaraceite, San Lorenzo, Arinaga ${ }^{5}$, Jinámar, etc. A modo de ejemplo, nos referiremos a las canteras de Hornos del Rey, en el pago de Jinámar, donde a principios del siglo XVII se registraba un centenar de hornos, que facilitaron material para la reconstrucción de la ciudad de Las Palmas y sus fortalezas tras el ataque de Van der Does (Pérez Hidalgo 2012). Tal vez sea interesante recordar que en estas canteras, de naturaleza travertínica (Montelongo Parada 2013), se encontraron algunas vetas de especial calidad, usadas en 1711 para labrar cuatro pilas ornamentadas destinadas a la catedral de Santa $\mathrm{Ana}^{6}$.

A la luz de investigaciones geológicas recientes, entendemos que algunos de los materiales carbonáticos de Gran Canaria, de los que se suelen denominar caliches, podrían ser tobas y travertinos de edad relativamente reciente, depositados por surgencias de aguas carbonatadas de tipo similar a las que son objeto de estudio en la presente investigación. Para llegar a correlacionar con los travertinos de Gran Canaria los líticos que vemos depositados por el agua de las galerías, han sido fundamentales los trabajos publicados por Ana María Alonso Zarza y su equipo, especialmente dos de ellos: «Las tobas/travertinos del barranco de Calabozo: Un ejemplo de construcción rápida de un edificio carbonático alimentado por una tubería de regadío» (Alonso Zarza et al. 2012) y «Petrología, sedimentología y geoquímica

${ }^{4}$ Viera y Clavijo (1731-1813) anotaba el uso de los confites (denominación que se da localmente a los esqueletos fósiles de rodolitos: algas coralinas de la especie florideophyceae) de La Isleta (Gran Canaria) para fabricación de cal, sobre la que informa que es excelente para el blanqueo de las casas y una buena materia adsorbente para la medicina. Los rodolitos, denominados localmente "confites», "cotufas», etc., los podemos encontrar actualmente en varios puntos del archipiélago: algunas playas de la zona nordeste de Fuerteventura se conforman casi íntegramente de este material; en Punta del Hidalgo (Tenerife) podemos ver rodolitos aún coloreados, en las charcas someras, siendo abundantes los restos blanqueados que se integran con la arena basáltica de la costa. Los confites de La Isleta son objeto en estos momentos de estudio científico por parte del grupo BIOCON de la ULPGC.

Museo de la Cal.

5 Los hornos de cal de Arinaga ubicados en el paseo marítimo se han rehabilitado como

${ }^{6}$ Los historiadores suelen anotar que estas pilas están realizadas en «mármol de Jinámar». Tres de ellas permanecen en la catedral de Santa Ana, una se expone actualmente en la Casa de Colón. Para información más detallada sobre este tema puede verse el artículo de Sánchez Bonilla (2018, 151-168). 
de los travertinos y tobas del Barranco de Azuaje (Gran Canaria): Características e implicaciones» (Rodríguez Berriguete 2017).

Como último elemento a destacar dentro de esta visión panorámica de las aguas carbonatadas y cales de Canarias, anotaremos a continuación un par de detalles históricos relativos a Tenerife. En primer lugar destacaremos que Viera y Clavijo (1731-1813) hizo referencia a las caleras de La Rambla (Rambla de Castro, Los Realejos, Tenerife), comentando la fama debida a sus bellas petrificaciones y la excelente blancura de su cal; información que encuentra mayor detalle en la ficha n. ${ }^{\circ} 15$ del Catálogo inventario de yacimientos paleontológicos de Santa Cruz de Tenerife, donde se especifica que estas costras calcáreas contienen impresiones y restos fosilizados vegetales y su origen está relacionado con nacientes de aguas calcáreas, posiblemente calientes, que depositaron el contenido en $\mathrm{CO}_{3} \mathrm{Ca}$ sobre las hojas, ramas y frutos de los vegetales que se encontraban en el suelo (García-Talavera Casañas, Paredes Gil y Martín Oval 1989).

Referencia de enorme interés en relación con nuestra investigación es una anotación sobre fabricación de cal en los Altos de Arico, que incluye Fernando Sabaté Bel en su tesis doctoral:

En la década de los años cuarenta, en un contexto que apuntaba hacia la intensificación, se construyó en Arico un ingenio calero protoindustrial [...] ubicado nada menos que en la zona alta de El Bueno, a más de mil metros de altitud, y en un paraje alejado de cualquier vía rodada de comunicación [...]. Ya se había observado que las aguas de algunas galerías de Arico portaban en disolución una gran cantidad de sales carbonatadas, producto de su mezcla en el acuífero con gases carbónicos vinculados a la historia volcánica de la Isla. Estas sales, además de comprometer la propia calidad del agua de riego, suponían -y siguen suponiendo en la actualidadun problema técnico grave: al entrar en contacto con el aire, se precipitan y van formando costras calcáreas cada vez más gruesas que, si no se limpian de manera periódica, pueden acabar colmatando las conducciones. La demanda de cal y su presencia potencial en el agua que brotaba en la parte alta se fusionaron por medio de un ingenioso procedimiento: el agua se hacía pasar a través de una piscina dividida en compartimentos (a modo de serpentín) para frenar su velocidad y favorecer que las concreciones calcáreas se fueran depositando en el fondo de la cubeta. De allí se recogían, suministrando la materia prima (carbonato cálcico), que procedía a transformarse en cal (óxido de calcio) en un horno de gran tamaño situado un poco más abajo. Se explica así por qué los enclaves humanizados situados en las inmediaciones de ese horno (grupos de cuevas, hornos de pasar fruta, lajas impermeabilizadas para recoger agua de lluvia, pavimento de alguna era) exhiben un empleo abundante de la cal, superior desde luego a la moderación con que ésta aparece en otros ámbitos del medio rural del Sur. En fin: la lógica de esta pequeña y 'astuta' infraestructura se puede sintetizar del siguiente modo: el propio residuo que transporta el agua (las sales carbonatadas que la contaminan), se logra transformar en un recurso (la cal como material de construcción), que va a servir precisamente para construir los medios que transportan esa misma agua (Sabaté Bel 2011, 289-290).

Contamos, por tanto, con la posibilidad de analizar el resultado que, tras casi ocho décadas, muestran las construcciones en que se aplicaron aquellas cales, 


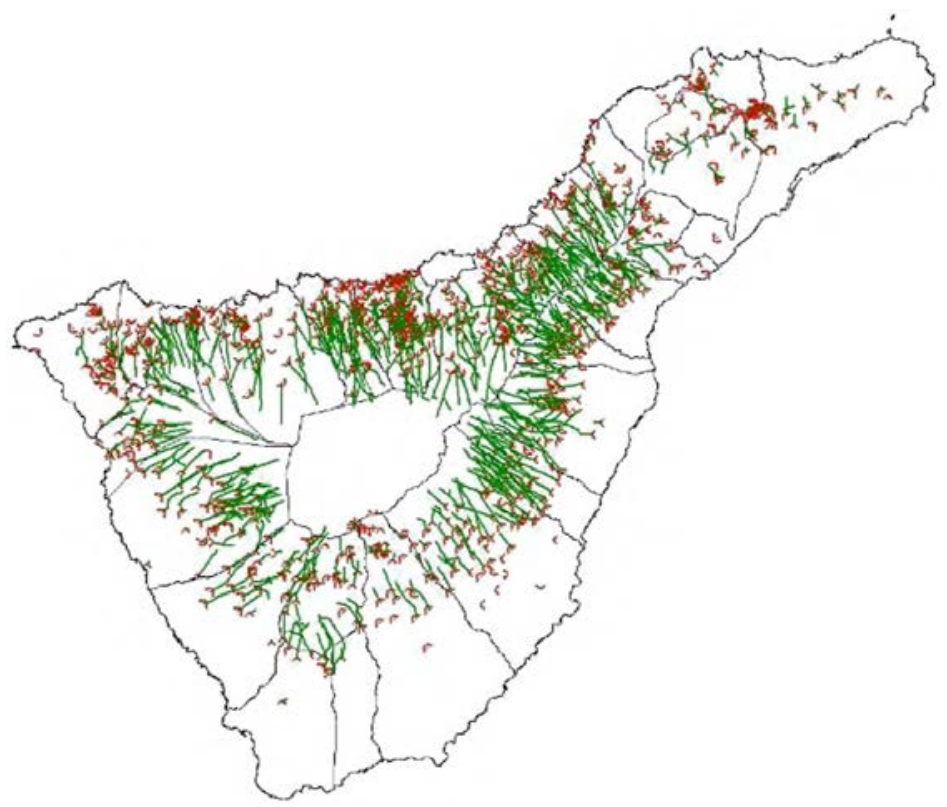

Figura 1. Mapa de galerías de agua de Tenerife. Desde www.aguastenerife.org es posible llegar a la ficha de cada una de las galerías. Para cada una de ellas ofrece datos relativos a la ubicación, trayectoria y longitud de la perforación, evolución de caudales en las últimas décadas, peligrosidad (presencia de $\mathrm{CO}_{2}$ ) en el interior de la mina, $\mathrm{y}$, para las galerías activas, datos químicos del agua.

fabricadas con los sedimentos depositados por el agua de galerías de la misma zona en que se ubica una de las seleccionadas para el trabajo experimental de nuestro proyecto de investigación, la galería El Rebosadero.

\section{DESARROLLO EXPERIMENTAL}

Partiendo del estado de la cuestión descrito, decidimos, por las razones ya anotadas, que el desarrollo de la parte experimental se llevaría a cabo, para este primer proyecto, en la isla de Tenerife. Además de contar con amplia bibliografía general sobre el tema, el Consejo Insular de Aguas de Tenerife, CIAT, ofrece un mapa de altísimo interés en función de nuestros objetivos, mediante el que podemos acceder a datos actualizados de todas las galerías, incluso de las que ya no están en producción, e informes de gran interés sobre evolución de los acuíferos, entre los que cabe destacar «Evolución cuantitativa del sistema acuífero de Tenerife» (Farrujia, Braojos y Fernández 2006), trabajo que, tras analizar datos desde 1930, concluye que «las medidas realizadas demuestran que a corto y medio plazo los recursos de origen subterráneo continuarán descendiendo». 
El Plan Hidrológico de Tenerife deja constancia de la gran preocupación que genera el estado químico del acuífero de las Cañadas ${ }^{7}$ y de manera especial las aguas de la comarca hidráulica de la vertiente norte, la de mayor caudal, cuyas aguas «son de mala calidad, con altos contenidos en iones fluoruro, bicarbonato y sodio». Alude a problemas similares de conductividad en la zona sureste; ante este panorama propone la búsqueda de procedimientos de desalación que permitan reducir el flúor (adecuación a las exigencias técnico-sanitarias) y los bicarbonatos (para garantizar el uso agrícola, especialmente el del plátano, muy sensible ante niveles altos de conductividad), que se concretan en la instalación de tres EDAS mediante técnicas de ósmosis inversa, ya en funcionamiento en La Guancha, Icod y Guía de Isora, cuyas salmueras de desecho actualmente son conducidas a pozos cercanos a la costa. Estamos dando pasos para intentar conformar un grupo multidisciplinar capaz de buscar utilidad a estas salmueras «residuo».

TABLA 1. HIDROQUÍMICA CORRESPONDIENTE A LAS SALMUERAS DE LAS TRES EDAS ACTUALMENTE ACTIVAS EN TENERIFE. DATOS DE ANALÍTICAS LLEVADAS A CABO EN JUNIO DE 2018, FACILITADOS POR EL CONSEJO INSULAR DE AGUAS DE TENERIFE

\begin{tabular}{|c|c|c|c|}
\hline & EDAS CRUZ de TARIFE & EDAS ICOD & EDAS Aripe \\
\hline \multicolumn{4}{|l|}{ Datos de campo } \\
\hline - Conductividad (O2mg/l) & 11700.00 & 11080.00 & 110600.00 \\
\hline - Temperatura agua $\left({ }^{\circ} \mathrm{C}\right)$ & $17^{\prime} 70$ & $18 ’ 40$ & $18^{\prime} 10$ \\
\hline \multicolumn{4}{|l|}{ Análisis: } \\
\hline$-\mathrm{PH}$ & 7.80 & 7.60 & 8.10 \\
\hline - Conductividad & 11570.00 & 10940.00 & 10280.00 \\
\hline$-\mathrm{SiO} 2(\mathrm{mg} / \mathrm{l})$ & 43 & 49 & 46 \\
\hline - Alcalinidad TAC (oF) & 725.49 & 680.16 & 593.93 \\
\hline - Dureza total $\left({ }^{\circ} \mathrm{F}\right)$ & 276.44 & 265.27 & 326.02 \\
\hline \multicolumn{4}{|l|}{ Cationes } \\
\hline$-\mathrm{Ca}(\mathrm{meq} / \mathrm{l}-\mathrm{mg} / \mathrm{l})$ & $8.18-164.00$ & $7.78-156.00$ & $16.91-359.00$ \\
\hline$-\mathrm{Mg}(\mathrm{meq} / \mathrm{l}-\mathrm{mg} / \mathrm{l})$ & $46.46-565.00$ & $46.65-543.00$ & $46.00-567.00$ \\
\hline$-\mathrm{K}(\mathrm{meq} / \mathrm{l}-\mathrm{mg} / \mathrm{l})$ & $16.50-645.00$ & $13.76-538.00$ & $11.13-435.00$ \\
\hline$-\mathrm{Na}(\mathrm{meq} / \mathrm{l}-\mathrm{mg} / \mathrm{l})$ & - & $93.43-2149.00$ & $79.13-1820.00$ \\
\hline \multicolumn{4}{|l|}{ Aniones } \\
\hline - CO3 (meq/l - mg/l) & - & - & - \\
\hline - HCO3 (meq/l - mg/l) & $145.07-8851.00$ & $136.00-8298.00$ & $118.77-7246.00$ \\
\hline - SO4 (meq/l - mg/l) & $18.57-892.00$ & $18.20-874.00$ & $30.71-1475.00$ \\
\hline
\end{tabular}

7 Para mayor información sobre las circunstancias que subyacen en la evolución de este acuífero, puede verse la tesis doctoral de Rayco Marrero (2010). 


\begin{tabular}{lccc}
\hline - CL $(\mathrm{meq} / \mathrm{l}-\mathrm{mg} / \mathrm{l})$ & $5.98-212.00$ & $4.40-156.00$ & $6.29-223.00$ \\
\hline - NO3 $(\mathrm{meq} / \mathrm{l}-\mathrm{mg} / \mathrm{l})$ & $0.85-52.80$ & $0.56-34.60$ & $0.31-19.20$ \\
\hline - NO2 (meq/l - mg/l) & $0.00-0.10$ & $0.00-<0.10$ & $0.00-<0.10$ \\
\hline - PO4 (meq/l - mg/l) & $0.33-10.50$ & $0.25-7.90$ & $0.27-8.50$ \\
\hline Total sólidos disueltos $(\mathrm{g} / \mathrm{l})$ & 13,80 & 12,81 & 12,20 \\
\hline
\end{tabular}

Los trabajos de campo realizados por nuestro GI en diversas zonas de Tenerife han permitido obtener un importante conjunto de muestras de agua y piedra. A modo de ejemplo se incluyen a continuación algunas imágenes correspondientes tanto a los trabajos de campo para búsqueda de muestras como a su preparación para realizar las correspondientes analíticas.

A partir de las muestras obtenidas en diversas zonas de la isla (La Orotava, Los Realejos, Los Silos, La Guancha, Icod, Santiago del Teide, Guía de Isora, Arico, Arafo, etc.), se inicia el estudio de los depósitos carbonáticos procedentes tanto de su acumulación sobre elementos del entorno (ramas, pinocha, tubos de riego, etc.) como del interior de las tuberías y/o canales (abiertos o cerrados) de conducción de estas aguas naturales procedentes de galerías; con el objetivo concreto de determinar la composición química y características físicas macroestructurales de los estratos observados en el depósito.

La metodología usada ha consistido en la realización de:

1. ${ }^{\circ}$ Un estudio de microscopía electrónica con microsonda EDX de dos tipos de muestras procedentes de estos depósitos, a saber:

a. Muestras en polvo procedentes de la limadura de los contenidos de los diferentes estratos realizadas longitudinalmente a las piezas de los depósitos. Con este estudio se determina la composición elemental de las muestras en polvo ( $\%$ atómico de cada elemento químico detectado).

b. Estratos observados en un corte transversal a la pieza de depósito recogida en el yacimiento. Lo que permite determinar también la composición elemental de las sustancias presentes en cada estrato y la microestructura (tipos de granos observados por SEM).

Las composiciones elementales de las muestras «b» deben coincidir globalmente con las de las muestras «a», y partiendo de ellas se puede hacer una primera asignación a los compuestos a los que probablemente correspondan.

2. ${ }^{\circ}$ Un estudio de difracción de rayos $\mathrm{X}$ de las muestras «b» anteriores, para determinar las especies cristalinas presentes en cada estrato, cuyos picos confrontamos con los difractogramas de especies cristalinas patrón (programas SCORE y MATCH 3 y bases de datos en sistema abierto). Por exclusión se determinan también los compuestos químicos no cristalinos presentes en cada estrato. 



Figura 2. a-c: panorama (cerca de galería Hoya de Leña, nov. 2018); d: muestra de interior canal de plástico; e: de interior c. hierro; f: de interior c. cemento; g: de exterior tubo plástico; i-j cortes transversales; k-m obtención de muestras en polvo.

Para finalizar la descripción de la parte analítica, anotemos un ejemplo: tomando como base las muestras SG1 a SG5 (sucesivos estratos sedimentados exteriormente sobre tubo de plástico de un punto de recogida cercano a la bocamina de la galería Salto del Guanche, en Guía de Isora), puede afirmarse que estamos dentro del campo de materiales estructuralmente calizos. La formación de estratos podría ser estacional, ya que se observan coincidencias de estructura y composición química entre capas alternas que también visualmente presentan color y textura simila- 

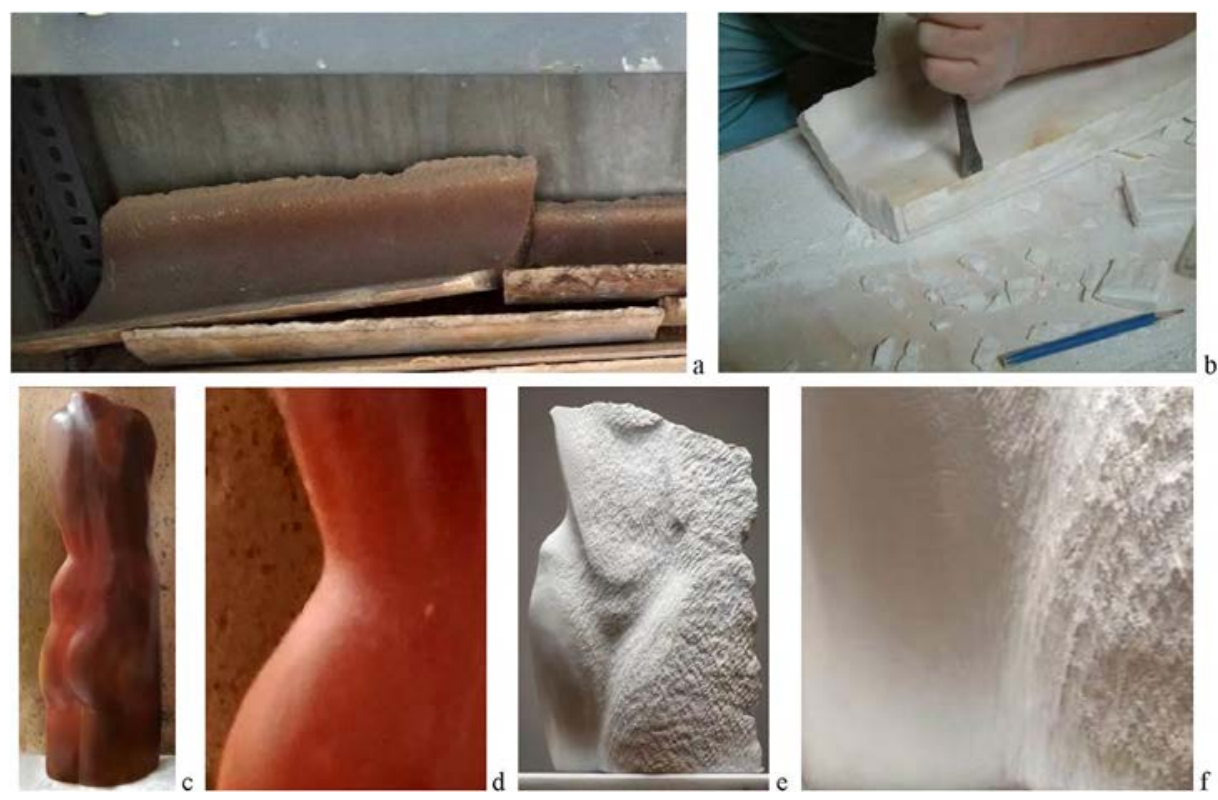

Figura 3. Materiales de partida, a: de canal de plástico, galería Añavingo-Arafo, b: de canal de hormigón, galería El Rebosadero-Arico), c-f: transformación mediante procesos escultóricos de desbaste, labra y pulimento.

res. Una primera aproximación analítica sobre la base de datos SEM/EDX y DRX ${ }^{8}$ (fig. $2 \mathrm{i}$ y fig. $2 \mathrm{k}$ ) muestra la presencia mayoritaria de aragonito en los estratos analizados con presencia de calcita (9: 1), en alguno de ellos, de tonalidad diferenciada; se muestra la presencia de trazas de algún tipo de óxidos de hierro no cristalinos y de algún tipo de dolomita en vez de calcita.

Parte muy significativa del desarrollo experimental ha sido la ejecución de obras escultóricas mediante procesos de labra. Estamos preparando una exposición, con intención de que sea itinerante, en salas de arte de diversas zonas (las que presentan aguas con mayor capacidad petrificante). Con esta exposición se pretende mostrar la belleza de los materiales y sus posibilidades como recurso escultórico, que también podría serlo artesanal.

Se ha llevado a cabo también una labor muy significativa en la preparación de moldes y madreformas para petrificación, con diversos materiales y técnicas, dando una importancia singular a la preparación de moldes mediante programas de modelado 3D.

${ }^{8}$ Datos experimentales registrados en los servicios SIDIX y SME del SEGAI-ULL. 

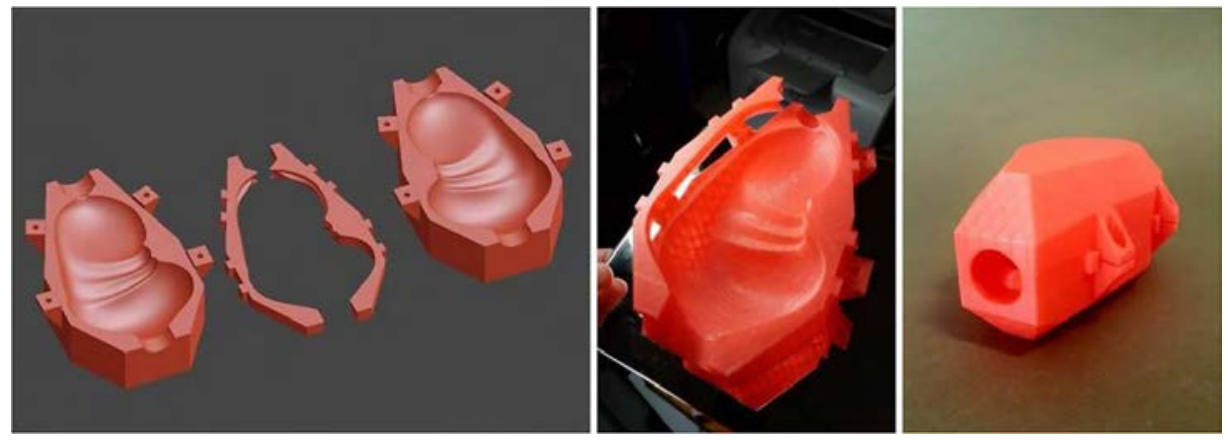

Figura 4. Molde a piezas (reutilizable). La construcción y el despiece de la forma se llevaron a cabo con software Blender.
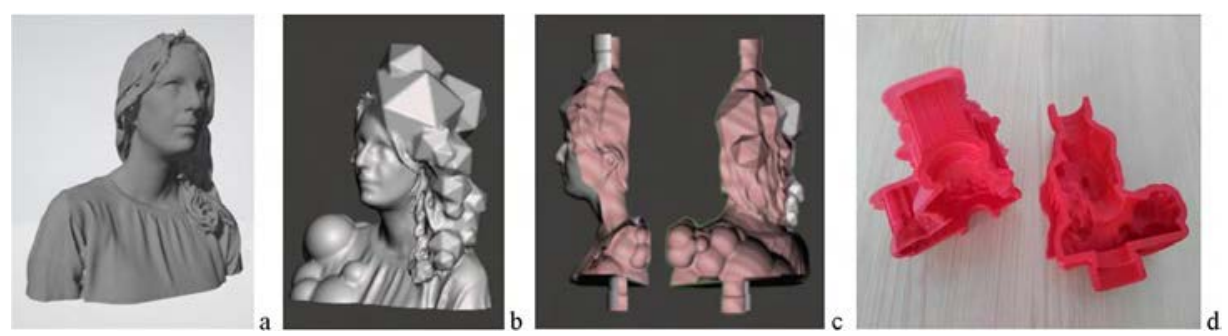

Figura 5. Molde obtenido mediante técnica mixta: escaneo 3D de un busto humano (escáner portable 3DSense). Edición mediante programa de modelado 3D y transformación creativa, aplicando operaciones de offset y sustracción booleana se obtiene la forma negativa / molde, que ya lleva incorporados los tubos de entrada/salida del agua y conexión con otros moldes.

Estos moldes cerrados tienen como objetivo la petrificación interna; sumados unos a otros, conformarán tuberías a través de las cuales se hará pasar el agua para que deposite las rocas carbonáticas según las formas dadas. Además de los moldes 3D, también se usarán moldes realizados con materiales tradicionales (escayola, silicona, etc.). Nos encontramos en proceso de producción masiva de moldes e identificación de los mejores lugares para su instalación. Como precedente en que se basa la viabilidad de esta técnica contamos con el TFM de Francisco Cordeiro (2015), realizado en colaboración con el Grupo de Investigación Arte y Entorno de la ULL.

Se ha planteado igualmente una experimentación amplia, conducente a la generación de formas escultóricas mediante moldes abiertos sobre los que transita el agua o mediante la inmersión de madreformas que propicien y ordenen la acumulación de sedimentos calcáreos de acuerdo con formas preestablecidas. Nos encontramos en proceso de producción masiva, con materiales diversos (alginator, cera, fibras naturales endurecidas con papilla de cal, etc.), cuidando siempre que esos materiales sean compatibles con el uso habitual del agua (consumo/regadío). Simultánea- 


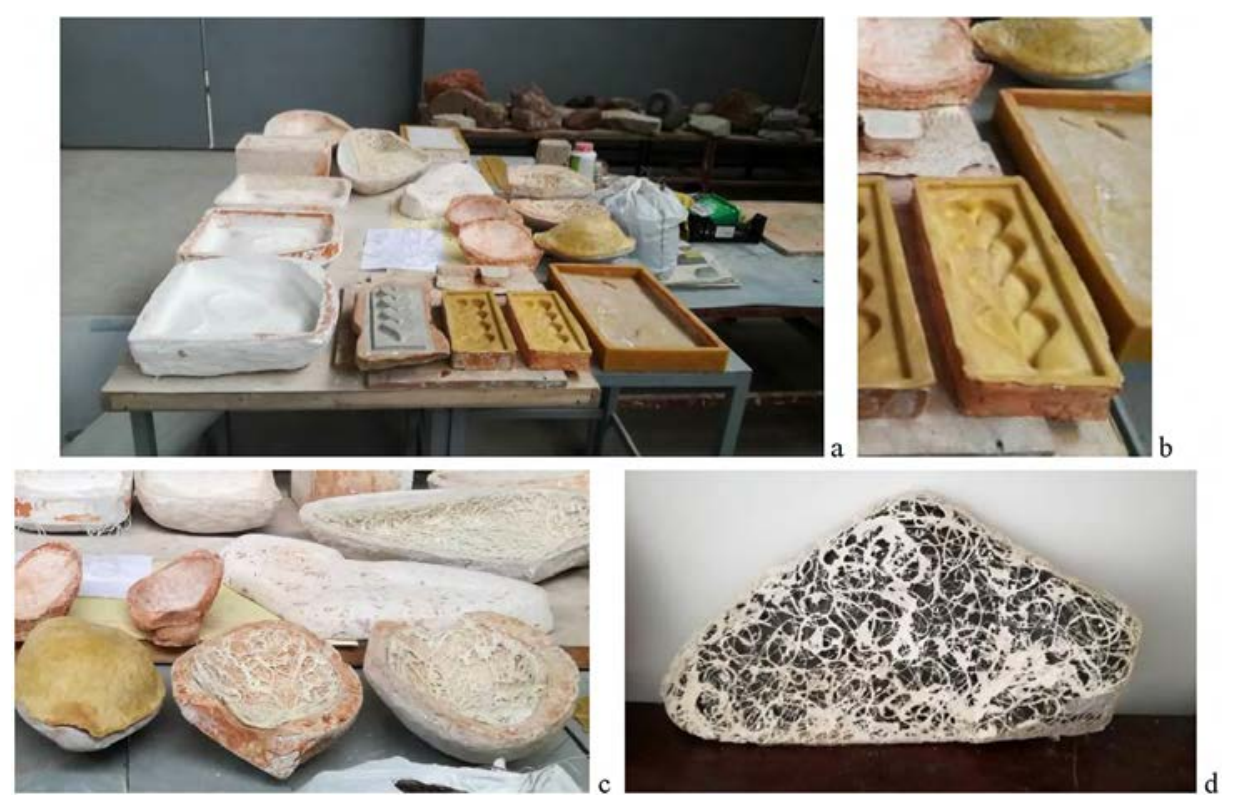

Figura 6. a-b: Moldes abiertos, c-d: madreformas, en diversos materiales: escayola, cera, silicona, alginator, fibras vegetales.

mente se están estableciendo acuerdos con diversas galerías de agua, con la idea de solicitar conjuntamente financiación competitiva para instalaciones.

\section{DISERTACIÓN FINAL}

Como se ha ido viendo, las islas Canarias conforman un entorno singular que, también en este campo, se puede convertir en laboratorio experimental desde el que analizar y poner en marcha proyectos cuyos protocolos serán, sin lugar a dudas, de gran interés para otras zonas que, debido al volcanismo residual, tienen aguas que presentan elevada conductividad y contenidos minerales que sobrepasan los autorizados por la normativa de abastecimiento vigente.

Estas aguas carbonatadas, que en Canarias se extraen del subsuelo mediante galerías/minas, al perder presión y temperatura e interaccionar con el aire depositan en su recorrido rocas carbonáticas, compuestas principalmente de aragonito y calcita, que por su consistencia, belleza y maleabilidad son de gran interés en el ámbito de los procesos escultóricos de tipo sustractivo. Pero, además, ofrecen enormes expectativas mediante procesos de petrificación en el interior de moldes o sobre madreformas, aspectos en los que ya se ha llegado a resultados alentadores, aunque requieren aún bastante desarrollo. 
Tanto en Canarias como en otros lugares con aguas de igual naturaleza, se está tendiendo a resolver el "problema» de la alta mineralización mediante plantas de tratamiento masivo del agua. Las más modernas y eficientes parecen ser las de tecnología basada en ósmosis inversa. Las plantas de tratamiento mediante ósmosis inversa llevan asociado, en general, un elevado consumo de energías fósiles, aunque, en las islas Canarias orientales, nuevamente pioneras en relación con el agua, se cuenta ya con ejemplos que se nutren de energía eólica. Además del consumo energético, está el problema de qué hacer con las salmueras residuales o con los subproductos derivados de la limpieza de membranas, y no conocemos ningún caso en que estos aspectos se estén resolviendo con un criterio medioambiental estricto, por lo que consideramos urgente la constitución de grupos multidisciplinares de investigación, con el objetivo de buscar alternativas desde una visión más acorde con la necesaria sostenibilidad.

Esta investigación la presentamos tras cerrar el primer proyecto financiado, que nos ha permitido avanzar bastante, aunque todavía estamos lejos de poder llegar a conclusiones definitivas. Nuestra intención es continuar trabajando para contar los resultados de los diferentes ensayos de petrificación con agua de galerías, y todavía no sabemos con seguridad si también con salmueras de rechazo. Esta investigación, que está aún en edad de crecer, quiere avanzar con miras amplias y de manera atrevida, por lo que está abierta a colaborar con otros investigadores e investigadoras de cualquier ámbito que tengan interés en "fuentes petrificantes».

Antes de concluir, queremos dejar anotado que este proyecto es «oficialmente» de los autores que aparecen en el encabezado, pero son muchos más los colaboradores. Merecen especial mención los geólogos Ramón Casillas, Ana M. ${ }^{a}$ Alonso Zarza y Nora Cabaleri, que han compartido con nosotros unos trabajos de campo inolvidables; Luis Enrique Hernández nos ha acogido, como siempre, en el Laboratorio de Calidad en la Construcción, dando pie a conversaciones de gran interés sobre las características excepcionales del entorno volcánico y sobre geotécnia. Dejamos constancia asimismo de nuestro más sincero agradecimiento, a D. Manuel Martínez, consejero del Consejo Insular de Aguas de Tenerife, y a Dña. Isabel Farrujia, técnico responsable de recursos subterráneos de dicha entidad y, con seguridad, una de las personas que mejor conoce las especificidades del agua en nuestra isla. 


\section{BIBLIOGRAFÍA}

Alonso Zarza, Ana María, Álvaro Rodríguez Berriguete, María del Carmen Cabrera, Alfonso Meléndez Hevia y L.F. Martín. 2012. «Las tobas/travertinos del barranco de Calabozo: Un ejemplo de construcción rápida de un edificio carbonático alimentado por una tubería de regadío", Geotemas 13: 44-47, https://eprints.ucm.es/55612/1/Alonso-Zarzaetal2012tufaCalabozo1.pdf (consultado el 6 de mayo de 2019).

Cordeiro Delgado, Francisco Bernardo. 2015. Aplicación del proceso de petrificación calcárea al diseño de souvenirs. Trabajo de fin de máster. Universidad de La Laguna. https://riull.ull. es/xmlui/handle/915/7160 (consultado el 6 de mayo de 2019).

Consejo Insular de Aguas de Tenerife. n.d. «Obras de captación de aguas subterráneas». https:// www.aguastenerife.org/ (consultada el 6 de mayo de 2019).

Farrujia de la Rosa, Isabel, Juan José Braojos Ruis y José D. Fernández Bethencourt. 2006. «Evolución cuantitativa del sistema acuífero de Tenerife», en III Congreso de Ingeniería Civil y Medio Ambiente. Agua, Biodiversidad e Ingeniería, Zaragoza, 25-27 de octubre de 2006. https://www.aguastenerife.org/index.php?option=com_content\&view=article\& id=157\&Itemid=1143 (consultado el 6 de mayo de 2019).

García-Talavera Casañas, Francisco, Rafael Paredes Gil y Mercedes Martín Oval. 1989. Catálogo-inventario yacimientos paleontológicos: provincia de Santa Cruz de Tenerife. La Laguna: Instituto de Estudios Canarios.

Hausen, Hans. 1958. "Contribución al conocimiento de las formas sedimentarias de Fuerteventura (Islas Canarias)", Anuario de Estudios Atlánticos 4: 1-84.

Hernández Delgado, Francisco y María Dolores Rodríguez Armas. 1993. «Hornos de cal (caleras) en Lanzarote", Aguayro 204 (julio): 15-18.

Marrero DíAz, Rayco. 2010. «Modelo hidrogeoquímico del acuífero de las cañadas del Teide, Tenerife, Islas Canarias». Tesis doctoral. Universidad Politécnica de Cataluña.

Pérez Hidalgo, Humberto Manuel. 2010. Origen y noticias de lugares de Gran Canaria. Las Palmas de Gran Canaria: Fundación Mapfre Guanarteme.

Rodríguez Berriguete, Álvaro. 2017. «Petrología, sedimentología y geoquímica de los travertinos y tobas del Barranco de Azuaje (Gran Canaria): características e implicaciones». Tesis doctoral. Universidad Complutense de Madrid.

SÁnchez Bonilla, María Isabel. 2015. «Las piedras del agua. Posibilidades escultóricas y propuesta ambiental», en V Simposio Virtual Internacional Valor y Sugestión del Patrimonio Artístico y Cultural, Universidad de Málaga, 8 a 22 de julio de 2015. Acceso al pre-print: https:// riull.ull.es/xmlui/handle/915/7158 (consultado el 6 de mayo de 2019).

SÁnchez Bonilla, María Isabel. 2018. «Pilas para agua bendita del siglo xvin realizadas en piedra de Jinámar", Anales de la Real Academia de Bellas ArtesSan Miguel Arcángel 11: 151-168.

Sabaté Bel, Fernando. 2011. El país del pargo salado. Naturaleza, cultura y territorio en el Sur de Tenerife (1875-1950). La Laguna: Instituto de Estudios Canarios.

Viera y Clavijo, José de. 1942. Diccionario de historia natural de las Islas Canarias o Índice alfabético, descripción de sus tres reinos animal, vegetal y mineral. Santa Cruz de Tenerife: Imprenta Valentín Sanz. 
